\title{
Hepatic Vein Microdialysis is not Equivalent to Intrahepatic Microdialysis Monitoring for the Detection of Metabolic Changes Induced by Arterial Ischemia in a Pig Liver Model
}

D’Souza MA' ${ }^{1 *}$, Von Platen $A^{2}$, Rooyackers $0^{3}$ and Nowak $\mathrm{G}^{2}$

${ }^{1}$ Division of Surgery, CLINTEC, Karolinska University Hospital, Sweden

${ }^{2}$ Division of Transplant Surgery, Department for Clinical Science, Intervention and Technology (CLINTEC), Karolinska Institute, Karolinska University Hospital, Sweden

${ }^{3}$ Department of Anaesthesiology and Intensive Care, Karolinska Institute, Karolinska University Hospital, Sweden

\section{Research article}

Volume 5 Issue 1

Received Date: March 17, 2021

Published Date: April 01, 2021

DOI: $10.23880 /$ ijtps-16000158

*Corresponding author: Melroy A D’Souza MS, DNB, MRCSEd, Division of Surgery, CLINTEC, Karolinska Institute, Karolinska University Hospital, Huddinge, 141 86, Stockholm, Sweden, Tel: +46 8585 86019; Email: melroy.dsouza@sll.se

\section{Abstract}

Background: Intrahepatic (intraparenchymal) microdialysis is a well-established method used to monitor liver metabolism. A less invasive alternative would be to use a transjugular approach to place a microdialysis catheter in the middle hepatic vein and measure these metabolites intravenously in the liver. The aim was to study whether these two methods would be comparable for metabolic monitoring in a pig liver model.

Methods: Three microdialysis catheters with molecular cut-off $20 \mathrm{kDa}$ were inserted into the liver, middle hepatic vein and subcutaneously in 8 pigs used in the experiment. The flow rate was $0.3 \mu \mathrm{l} / \mathrm{min}$ in the intrahepatic and subcutaneous (reference) catheters and $1 \mu \mathrm{l} / \mathrm{min}$ in the hepatic vein catheter. During a 2-hour period of equilibration, a steady state was reached after 1 hour. The hepatic artery was then clamped for a period of 4 hours to induce ischemia. Dialysate samples were collected at 15-minute intervals from the hepatic and intravenous catheters and 30-minute intervals from the reference catheter and analysed for glucose, glycerol, lactate and pyruvate. The lactate/pyruvate ratio was calculated.

Results: Lactate and pyruvate levels in the intrahepatic and subcutaneous catheters showed significant changes after hepatic arterial clamping. Glycerol and the lactate/pyruvate ratio in the intrahepatic catheter showed increases, which were not significant, but spontaneously normalized despite persistent clamping. There were no changes in the metabolites measured by the hepatic vein catheter throughout the experiment.

Conclusion: Hepatic vein microdialysis cannot be used equally as compared to direct intrahepatic (intraparenchymal) placement regarding the monitoring of glucose, glycerol, lactate and pyruvate.

Keywords: Liver; Metabolism; Microdialysis; Ischemia

\section{Introduction}

Monitoring the metabolic and biochemical milieu of tissues has been achieved by the method of microdialysis, the principle of which is to mimic the passive function of a capillary blood vessel [1]. The microdialysis catheter has a double lumen and a semi-permeable dialysis membrane at the tip, which is inserted into the tissue. The catheter is 


\section{International Journal of Transplantation \& Plastic Surgery}

perfused with an isotonic fluid at a low velocity allowing molecules that are small enough, to cross the dialysis membrane by the process of passive diffusion. A process of equilibration occurs with the surrounding tissue into which the catheter is inserted and the fluid in the microdialysis catheter, now reflecting the composition of the surrounding extracellular fluid. The fluid in the catheter then returns via the inner lumen and can then be sampled in microvials (dialysate) at desired time intervals [2]. Microdialysis has been used for monitoring tissues and to detect complications before they become manifest or detectable in peripheral blood chemistry. This has led to application in many clinical scenarios and organs including brain, heart, muscle and subcutaneous tissue [3-6].

Microdialysis has been assessed for monitoring of hepatic metabolism in the setting of resection and transplantation [7-11]. Glucose and its metabolic products, lactate and pyruvate as well as glycerol can provide information about the metabolic state of the liver. Ischemic injury to the liver leads to significant alterations in metabolism and levels of these small molecules [2,7]. Glucose, lactate, and pyruvate have been used as markers of the oxidative status of a tissue and glycerol as a marker of cell membrane injury. Moreover, as an indicator of changes in the tissue's redox status, the lactate/pyruvate ratio $(\mathrm{L} / \mathrm{Pr})$ is calculated $[8,10$ 12]. Although a relatively invasive method, intrahepatic microdialysis is safe and has a low complication rate, despite the intraparenchymal placement of the catheter. It has however not achieved widespread clinical application and has not yet been introduced in the standard postoperative monitoring protocol after liver resection or transplantation. In the setting of liver transplantation, microdialysis has been used for up to 10 days post-transplantation in some studies to detect ischemic complications and rejection [9]. Most patients find the intrahepatic microdialysis catheter uncomfortable and there is also the inconvenience of being woken up at night for hourly sampling etc.

To circumvent these issues one approach would be to use an intravascular microdialysis catheter in the middle hepatic vein and measure these metabolites. This could be achieved through a transjugular access and would be a less invasive approach as compared to hepatic microdialysis in which placement of the catheter requires an open operation. Also, a venous catheter could be replaced when needed. The microdialysis catheter in the hepatic vein, samples blood directly as it leaves the liver, which conceptually should be close to the hepatic interstitial environment that is sampled by an intrahepatic catheter.

The aim of this study was to explore whether hepatic vein microdialysis could be used to monitor intrahepatic metabolism. To achieve this, intrahepatic (intraparenchymal) and hepatic vein microdialysis for small molecules such as glucose, glycerol, lactate and pyruvate were compared in a pig model with hepatic arterial ischemia.

\section{Methods}

\section{Anaesthesia and surgical procedure}

A total of 8 female pigs (Swedish Landrace/York- shire/ Hampshire) littermate pigs, with a body weight of 30 to 35 $\mathrm{kg}$, were used for the experiments. Before the operation, all animals were fasted for 24 hours with free access to water. Anaesthesia was induced by an intramuscular premedication of $12 \mathrm{mg} / \mathrm{kg}$ ketamine hydrochloride (Ketaminol Vet, Veterinaria AG, Zurich, Switzerland), $5 \mathrm{mg} /$ kg azaperone (Stresnil Vet, Janssen-Cilag Pharma, Wien, Austria), and $0.05 \mathrm{mg} / \mathrm{kg}$ atropine (Atropin, NM Pharma AB, Stockholm, Sweden). Midazolam (1-4 mg/kg; Alpharma AS, Oslo, Norway) was given intravenously prior to intubation (Mallinckrodt Medical, Ireland; inner diameter 7.0-7.5). The anesthesia was maintained by inhalation of $1: 2 \mathrm{O}_{2} / \mathrm{N}_{2} \mathrm{O}$ plus $0.5 \%$ to $1.5 \%$ halothane, which was complemented with fentanyl (Alpharma AS, Oslo, Norway) if necessary. A Ringer acetate intravenous infusion $(710 \mathrm{~mL} / \mathrm{kg} / \mathrm{hour})$ was given at $37^{\circ} \mathrm{C}$. Blood gas analysis (i-STAT, Abbott, Abbott Park, IL; EG7 cartridges), electrocardiogram, body temperature, and urine production were continuously recorded during the experiment. The body temperature was maintained at $38^{\circ} \mathrm{C}$ to $39^{\circ} \mathrm{C}$ with an external heating device as needed.

\section{Microdialysis}

Before the abdominal incision, the CMA 60 microdialysis catheter (CMA Microdialysis AB) with a $20-\mathrm{mm}$ shaft and a $30-\mathrm{mm}$ membrane was inserted just under the skin over the left pectoral area and fixed in position as a subcutaneous reference measure. A midline laparotomy was then performed and the intrahepatic microdialysis catheter was inserted into the middle lobe of the liver. The catheter was introduced into the liver with a steel cannula with a split catheter and sutured safely to liver parenchyma according to a method previously described by us [12]. The CMA 70 microdialysis catheter (CMA Microdialysis AB) with a 60$\mathrm{mm}$ shaft and a 30-mm membrane was used in the liver. The 67 IV microdialysis catheter (M Dialysis AB) with a 130-mm shaft and a $30-\mathrm{mm}$ membrane was introduced into the right internal jugular vein which was isolated using an open access. The tip of the catheter was guided to be placed in the middle hepatic vein and advanced till it stopped in the liver and could not be moved further. The position of the catheter was confirmed by palpation. After the 3 probes were inserted, the inlets of the tubings were connected to microinfusion pumps (CMA 106 microinjection pump; CMA Microdialysis AB, Stockholm, Sweden) and perfusion started. A Ringer acetate- 


\section{International Journal of Transplantation \& Plastic Surgery}

like solution, T1 (containing sodium $147 \mathrm{mmol} / \mathrm{L}$, potassium $4 \mathrm{mmol} / \mathrm{L}$, calcium $2.3 \mathrm{mmol} / \mathrm{L}$, osmolality $290 \mathrm{mmol} /$ $\mathrm{kg}, \mathrm{pH}$ 6; CMA Microdialysis AB, Stockholm, Sweden) was pumped through the catheters. The flow rate was $0.3 \mu \mathrm{l} / \mathrm{min}$ in the intrahepatic and subcutaneous catheters and $1 \mu \mathrm{l} / \mathrm{min}$ in the hepatic vein catheter.

During a 2-hour period of equilibration, a steady state was achieved after 1 hour of catheter placement. The hepatic arteries were dissected and prepared for occlusion and were clamped 1 hour later. This was done by isolation, ligation and division of the vessels. The arterial supply was clamped for a period of 4 hours to induce hepatic ischemia. Throughout the equilibration period and the clamping, dialysate samples were collected at 15-minute intervals from the intrahepatic and intravenous catheter. Samples were collected at 30-minute intervals from the subcutaneous catheter. The liver was not manipulated during the monitoring period. Dialysates were analysed for glucose, glycerol, lactate and pyruvate and the lactate/pyruvate ratio (L/Pr) was calculated. The samples were analysed using colorimetric methods with a CMA 600 microdialysis analyzer (CMA Microdialysis AB), a clinical chemistry analyzer using enzymatic reagents and colorimetric measurements immediately after sampling. At the end of the experiment the pigs were sacrificed by an overdose of anaesthesia. The samples after being analyzed for glucose, glycerol, lactate and pyruvate were discarded.

The study was approved by the local animal ethics committee at the Karolinska Institute. Animals received care in accordance with the Swedish regulations on animal experimentation. All operative procedures and handling of the microdialysis were done under clean but not sterile conditions.

\section{Statistics}

Data are presented as mean $+95 \%$ confidence intervals for each metabolite (Glucose, Lactate, Pyruvate, Glycerol and the L/Pr) and for each catheter (Intrahepatic, Hepatic vein and Subcutaneous). Results were compared using ANOVA for repeated measurements. In case of significant difference between groups over time, analyses were complemented with the Bonferroni post-hoc test. Statistical analyses were performed using Statistica 13.2 software program. P values $<0.05$ were considered statistically significant.

\section{Results}

There were no complications associated with the insertion or removal of the microdialysis catheters. The results are presented in the graphs presented in Figures 1-5. There were no differences over time in the glucose levels measured by all 3 catheters both before and after arterial clamping (Figure 1). Glycerol levels measured over time in each catheter showed no significant changes before and after arterial clamping (Figure 2). There were significant differences in lactate levels measured between the catheters $(\mathrm{p}<0.01)$. Lactate levels measured by the subcutaneous catheter between 60-120 minutes and in the intrahepatic catheter between 105-150 minutes after arterial clamping lactate were significantly higher as compared to before clamping (Figure 3).

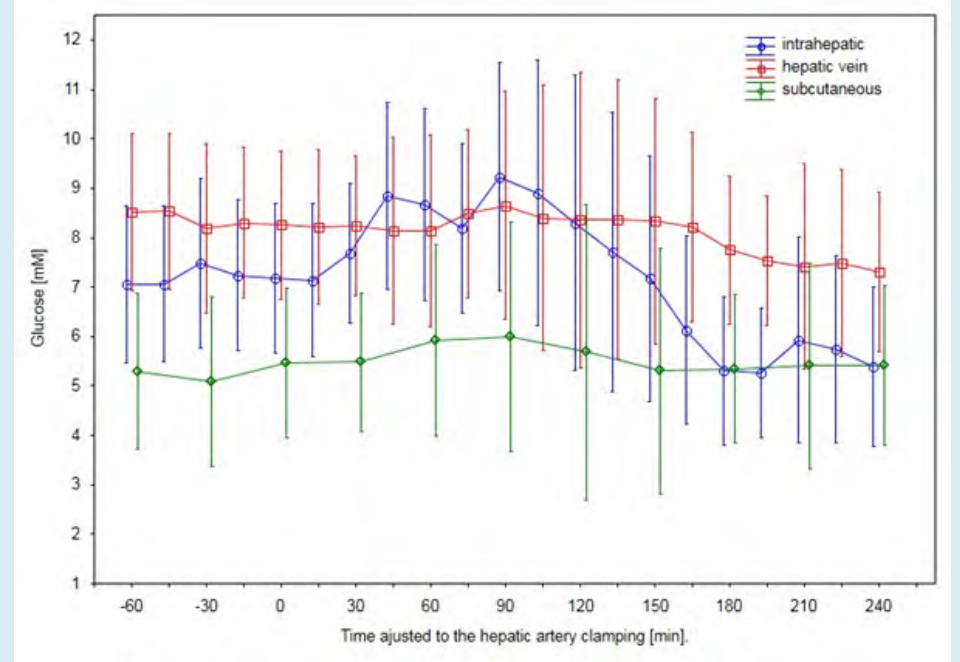

Figure 1: Glucose concentrations measured by the microdialysis catheters in the liver, hepatic vein and the subcutaneous tissue (mean $\pm 95 \% \mathrm{CI}$ ). There were no significant changes over time before and after arterial clamping and between the values measured by the catheters. 


\section{International Journal of Transplantation \& Plastic Surgery}

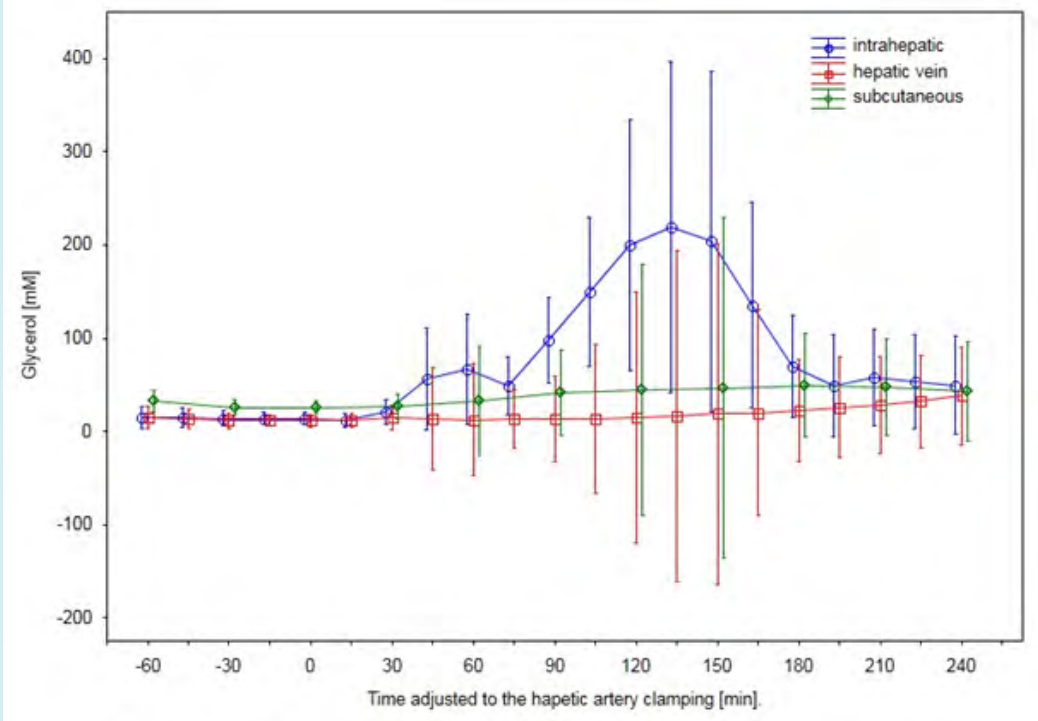

Figure 2: Glycerol concentrations measured by the microdialysis catheters showed no significant changes over time and between the 3 catheters (mean $\pm 95 \% \mathrm{CI}$ ).

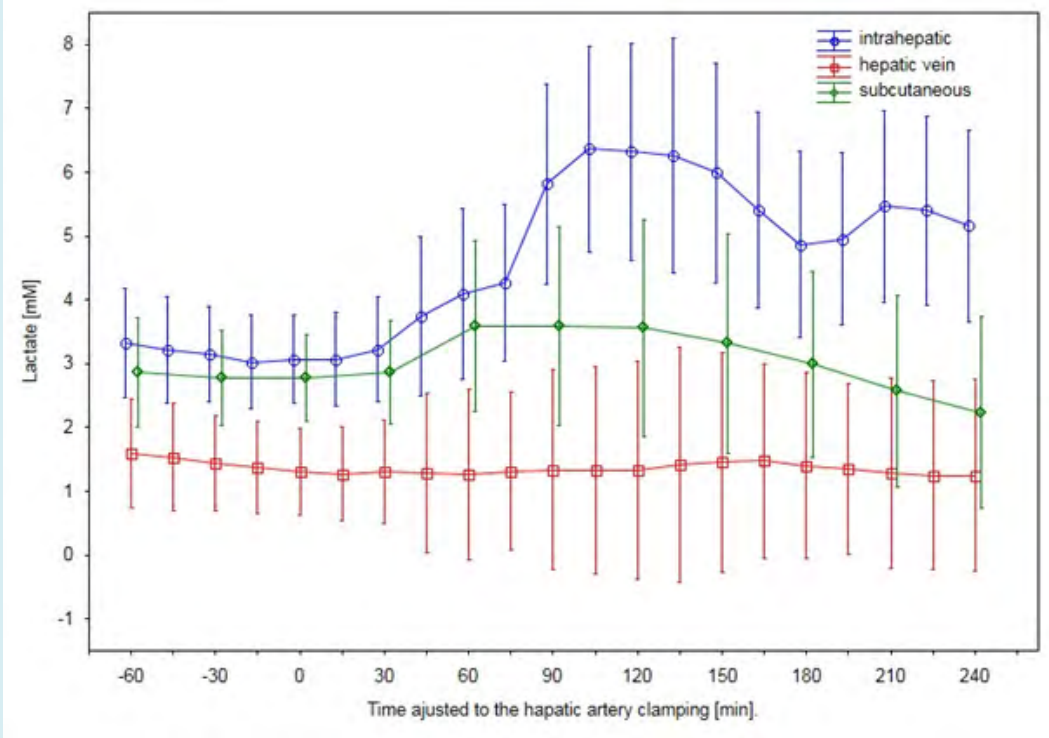

Figure 3: Lactate concentrations levels measured by the intrahepatic and the subcutaneous catheters increased significantly after arterial clamping with a time lag for this increase to be detected in the intrahepatic catheter (mean $\pm 95 \% \mathrm{CI}$ ). Lactate measured by the hepatic vein catheter showed no change over time.

There were significant differences between pyruvate levels measured in the different catheters $(p<0.001)$ with an increase in pyruvate levels subcutaneously between 60 120 minutes after arterial clamping $(\mathrm{p}<0.01)$. There was a decrease in pyruvate levels measured in the intrahepatic catheters from 90 minutes onwards after clamping $(\mathrm{p}<0.001)$ (Figure 4). There was no difference in the L/Pr measured by the 3 catheters (Figure 5). 


\section{International Journal of Transplantation \& Plastic Surgery}

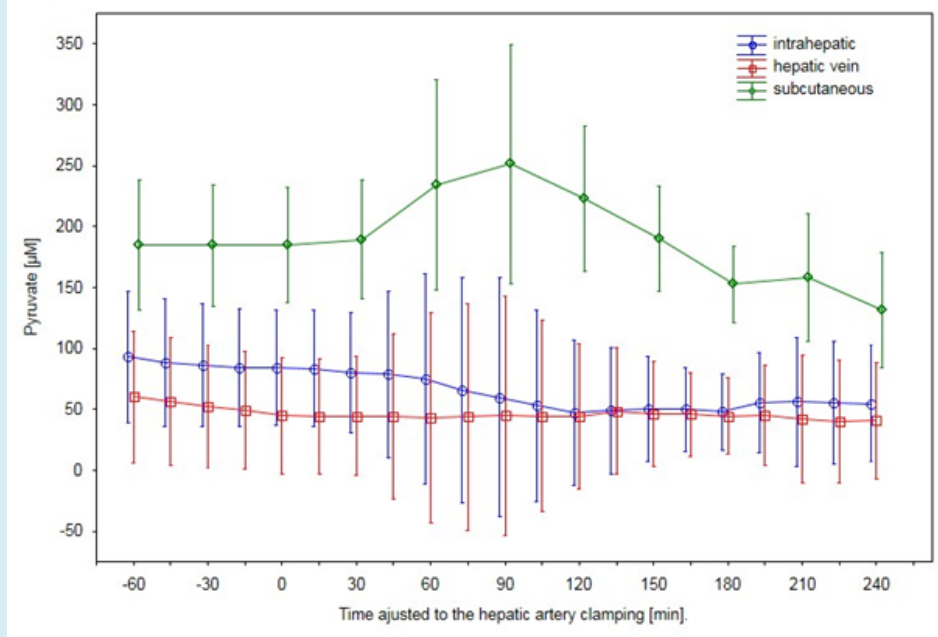

Figure 4: Pyruvate concentrations showed significant changes over time in the subcutaneous and intrahepatic catheters (mean $\pm 95 \%$ CI). Pyruvate levels increased subcutaneously between 60-120 minutes and decreased in the intrahepatic catheters from 90 minutes onwards after hepatic arterial clamping.

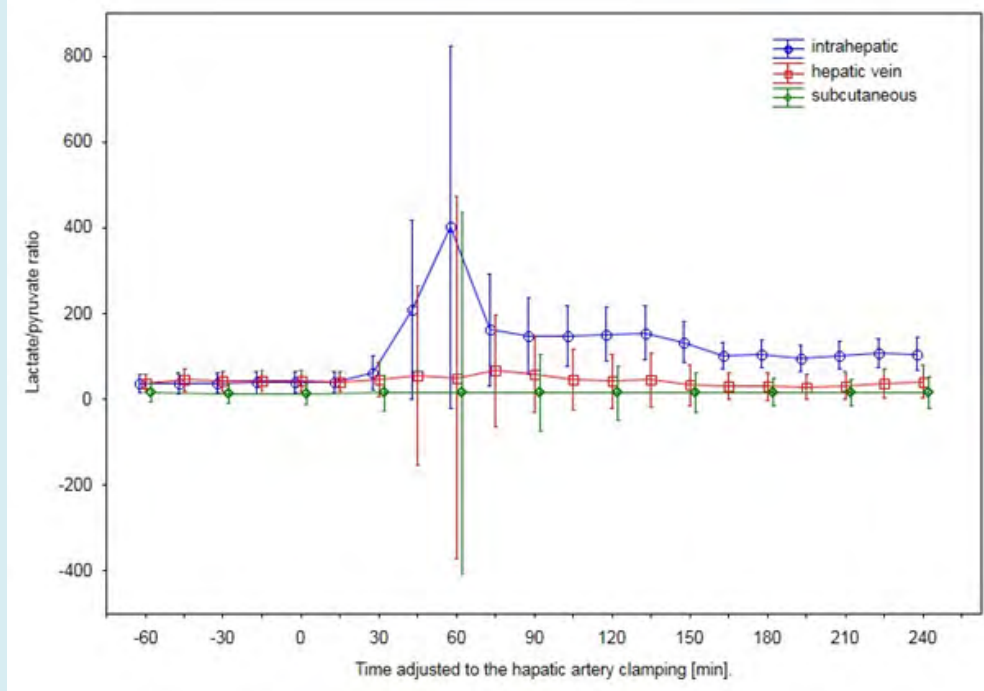

Figure 5: Calculated L/Pr for the 3 catheters showed no significant changes over time (mean $\pm 95 \% \mathrm{CI}$ ).

\section{Discussion}

Ischemia causes a shift of the intracellular metabolism from aerobic to anaerobic and ATP production can proceed only by the reduction of pyruvate to lactate. This results in an increase in lactate levels and a corresponding decrease in pyruvate resulting in an increase in the L/Pr [7]. Evaluation of our results shows that lactate levels measured by the intrahepatic and subcutaneous catheters increased significantly after hepatic arterial clamping (Figure 3). Our results show that glucose and glycerol levels and the $\mathrm{L} / \mathrm{Pr}$ increased in the intrahepatic catheters although the levels did not reach statistical significance (Figures 1,2,5). This could be due to the low number of pigs $(n=8)$ and the wide variation. Pyruvate levels measured by the intrahepatic catheter showed a significant decrease post-clamping (Figure 4). The findings of the present study are similar to results previously published by our group in a similar pig liver model of warm ischemia [13]. Like the findings of that study, glucose, lactate, glycerol levels and the L/Pr in the present study after an initial rise, continued to decline spontaneously despite persistent clamping. This could be because of a 


\section{International Journal of Transplantation \& Plastic Surgery}

possible increase in oxygen extraction by the hepatocytes from portal venous blood as we performed only a selective arterial clamping. What was novel in the present study is that pyruvate levels measured by the subcutaneous catheter showed a significant increase during the clamping phase while pyruvate levels declined in the liver. We speculate that this could suggest a systemic hypermetabolism as a response to the hepatic ischemia and could be part of a stress reaction.

The main aim of our study was to investigate whether microdialysis sampling of blood in the hepatic vein would reflect the metabolic environment and changes in the hepatic interstitial fluid directly sampled by an intrahepatic microdialysis catheter. Our results clearly show that none of the above changes in response to hepatic arterial clamping detected by the intrahepatic and subcutaneous catheters were registered in the hepatic vein catheter. Levels of glucose, glycerol, lactate and pyruvate and the calculated L/ Pr remained stable throughout the experimental period in the hepatic vein catheter.

Intravascular microdialysis has been used previously for the monitoring of glucose and lactate. This has been evaluated in both animal models and post-cardiac surgery and in the intensive care setting in humans [4,14-16]. These studies have demonstrated the technical feasibility of intravascular microdialysis. Results have shown good correlation between measurements made by the intravenously placed microdialysis catheter with arterial blood gas measurements for example [17]. Cannulating the hepatic vein is standard for procedures like TIPS (Transjugular Intrahepatic Portosystemic Shunt) creation and HVPG (Hepatic venous pressure gradient) measurement $[18,19]$. The usual route used for access is via the right or left internal jugular veins and this approach was used in our experiment. Intraoperative palpation was used to place the tip of the hepatic vein catheter as far into the liver as possible to be as close to the parenchyma. This control of catheter position in clinical practice can be evaluated using ultrasound examination. This was done to avoid the effect of dilution if the catheter membrane and tip was too far from the tissue of interest. Nonetheless, this could have played a factor in the results. Also, the hepatic venous outflow is a 'high flow' system and this could be a factor, which reduced the recovery of the metabolites. While this factor cannot be modified, increasing the membrane length could be one way to increase recovery. Another method would be to adjust the perfusion flow rate in the catheter to increase the recovery [20-22]. Catheter perfusion flow rates of $0.5,1$ and $2 \mu \mathrm{l} / \mathrm{min}$ have been used to optimize recovery with better results by decreasing the perfusion rate [20]. Disregarding the technical aspects of the study, it could also be that the composition of hepatic venous blood is not comparable to that of the hepatic interstitial environment (similar to the blood-brain barrier?) thus making this method unsuitable for hepatic metabolic monitoring.

In conclusion, our results show that while hepatic vein microdialysis is a conceptually attractive idea, in the present study it could not be used to represent direct intrahepatic microdialysis for detection and metabolic monitoring of changes induced by hepatic arterial ischemia, which cause significant injury and metabolic disturbances in the liver. Perhaps identification of other molecules or refinement of the technique may make it a feasible method in the future.

\section{Author Contribution}

Melroy A D'Souza: Analyzing of data and writing manuscript. Anna von Platen: Collection of data and writing manuscript.

Olav Rooyackers: Writing manuscript.

Greg Nowak: Study design, collection and analyzing of data and writing manuscript.

Grants and Financial Support: CMA Microdialysis AB, Stockholm, Sweden. Pregraduate School of Clinical Science, Karolinska Institute.

Disclosure: None of the authors have a financial interest in any of the products or devices mentioned in this report. None of authors declare conflicts of interest.

\section{References}

1. Ungerstedt U (1991) Microdialysis--principles and applications for studies in animals and man. J Intern Med 230(4): 365-373.

2. Sabroe JE, Ellebaek MB, Qvist N (2016) Intraabdominal microdialysis - methodological challenges. Scand J Clin Lab Invest 76(8): 671-677.

3. Hutchinson PJ, Jalloh I, Helmy A, Carpenter KL, Rostami E, et al. (2015) Consensus statement from the 2014 International Microdialysis Forum. Intensive Care Med 41(9): 1517-1528.

4. Gouezel C, Lorne E, Bonnet V, Fradin S, Saplacan V, et al. (2017) Assessment of changes in lactate concentration with intravascular microdialysis during high-risk cardiac surgery using the trend interchangeability method. Br J Anaesth 119(6): 1110-1117.

5. Ren G, Eiskjaer S, Kaspersen J, Christensen FB, Rasmussen S (2009) Microdialysis of paraspinal muscle in healthy volunteers and patients underwent posterior lumbar fusion surgery. Eur Spine J 18(11): 1604-1609.

6. Edsander-Nord A, Rojdmark J, Wickman M (2002) 


\section{International Journal of Transplantation \& Plastic Surgery}

Metabolism in pedicled and free TRAM flaps: a comparison using the microdialysis technique. Plast Reconstr Surg 109(2): 664-673.

7. Isaksson $B, D^{\prime}$ Souza MA, Jersenius $U$, Ungerstedt J, Lundell L, et al. (2011) Continuous assessment of intrahepatic metabolism by microdialysis during and after portal triad clamping. J Surg Res 169(2): 214-219.

8. Nowak G, Ungerstedt J, Wernerman J, Ungerstedt U, Ericzon BG (2002) Clinical experience in continuous graft monitoring with microdialysis early after liver transplantation. Br J Surg 89(9): 1169-1175.

9. Haugaa H, Thorgersen EB, Pharo A, Boberg KM, Foss A, et al. (2012) Early bedside detection of ischemia and rejection in liver transplants by microdialysis. Liver Transpl 18(7): 839-849.

10. Waelgaard L, Thorgersen EB, Line PD, Foss A, Mollnes TE, et al. (2008) Microdialysis monitoring of liver grafts by metabolic parameters, cytokine production, and complement activation. Transplantation 86(8): 10961103.

11. Silva MA, Murphy N, Richards DA, Wigmore SJ, Bramhall SR, et al. (2006) Interstitial lactic acidosis in the graft during organ harvest, cold storage, and reperfusion of human liver allografts predicts subsequent ischemia reperfusion injury. Transplantation 82(2): 227-233.

12. Nowak G, Ungerstedt J, Wernerman J, Ungerstedt U, Ericzon BG (2002) Metabolic changes in the liver graft monitored continuously with microdialysis during liver transplantation in a pig model. Liver Transpl 8(5): 424432.

13. Ungerstedt J, Nowak G, Ungerstedt U, Ericzon BG (2009) Microdialysis monitoring of porcine liver metabolism during warm ischemia with arterial and portal clamping. Liver Transpl 15(3): 280-286.

14. Schierenbeck F, Wallin M, Franco-Cereceda A, Liska J
(2014) Evaluation of intravascular microdialysis for continuous blood glucose monitoring in hypoglycemia: an animal model. J Diabetes Sci Technol 8(4): 839-844.

15. Rooyackers O, Blixt C, Mattsson P, Wernerman J (2010) Continuous glucose monitoring by intravenous microdialysis. Acta Anaesthesiol Scand 54(7): 841-847.

16. Lenkin PI, Smetkin AA, Hussain A, Lenkin AI, Paromov KV, et al. (2017) Continuous Monitoring of Lactate Using Intravascular Microdialysis in High-Risk Cardiac Surgery: A Prospective Observational Study. J Cardiothorac Vasc Anesth 31(1): 37-44.

17. Schierenbeck F, Nijsten MW, Franco-Cereceda A, Liska $\mathrm{J}$ (2014) Introducing intravascular microdialysis for continuous lactate monitoring in patients undergoing cardiac surgery: a prospective observational study. Crit Care 18(2): R56.

18. Keller FS, Farsad K, Rosch J (2016) The Transjugular Intrahepatic Portosystemic Shunt: Technique and Instruments. Tech Vasc Interv Radiol 19(1): 2-9.

19. Ferlitsch A, Bota S, Paternostro R, Reiberger T, Mandorfer $M$, et al. (2015) Evaluation of a new balloon occlusion catheter specifically designed for measurement of hepatic venous pressure gradient. Liver Int 35(9): 21152120.

20. Rooyackers O, Blixt C, Mattsson P, Wernerman J (2013) Continuous glucose monitoring by intravenous microdialysis: influence of membrane length and dialysis flow rate. Acta Anaesthesiol Scand 57(2): 214-219.

21. Wisniewski N, Torto N (2002) Optimisation of microdialysis sampling recovery by varying inner cannula geometry. Analyst 127(8): 1129-1134.

22. Hutchinson PJ, O'Connell MT, Al-Rawi PG, Maskell LB, Kett-White R, et al. (2000) Clinical cerebral microdialysis: a methodological study. J Neurosurg 93(1): 37-43. 